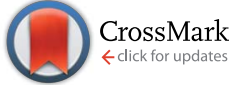

Cite this: RSC Adv., 2016, 6, 6651

\title{
Combining a flow reactor with spray dryer to allow the preparation of food-grade quality sodium 2- polyhydroxyalkyl-1,3-thiazolidine-4-carboxylates with a low environmental impact $\uparrow$
}

\begin{abstract}
Olalla Novo, ${ }^{a}$ Mercè Balcells, ${ }^{\text {ab }}$ Ramon Canela-Garayoa ${ }^{\text {ab }}$ and Jordi Eras*ab
The 1,3-thiazolidine-4-carboxylic acid (TCA) and 2-substituted 1,3-thiazolidine-4-carboxylic acids (2S-TCAs) are used as additive prodrugs of cysteine and glutathione in agronomy and in the food and pharmaceutical industries. Using a flow system coupled to a spray dryer, here we prepared the sodium salts of these compounds in powder form. The reaction was carried out using equimolar ratios of L-cysteine, aldehyde, and sodium hydroxide in water as solvent. Formaldehyde, three aldohexoses (D-glucose, D-galactose, D-mannose), three aldopentoses (D-xylose, D-ribose, Darabinose), and mixtures of these compounds were used as the starting aldehydes. We optimized the $\mathrm{pH}$, reaction temperature, and pump flow rate in order to reach conversions ranging from 55 to $100 \%$. The parameters for spray drying were optimized to reach yields over $79 \%$. Finally, the physical properties of the powders and the stability of these salts in water were measured and compared with those of the corresponding acids. The salts were found to be more stable than acids (13.6 to $17.7 \%$ vs. 59.8 to $92.1 \%$ to hydrolysis, respectively) after 30 days. As the reactants, the solvent, water, and the base used can be food-grade, the final product would meet the requirements for food-grade quality.
\end{abstract}

Received 25th September 2015 Accepted 7th January 2016

DOI: $10.1039 / c 5 r a 19880 a$

www.rsc.org/advances

\section{Introduction}

The 1,3-thiazolidine-4-carboxylic acid (TCA) and 2-substituted 1,3-thiazolidine-4-carboxylic acids (2S-TCAs) prepared from the condensation of cysteine and aldehydes have been studied extensively. ${ }^{1-4}$ The formation of a thiazolidine ring results from hemimercaptal formation from aldehydes on cysteine, followed by dehydration and cyclization. These compounds can be used as prodrugs of $\mathrm{L}$-cysteine, which can release the sulfhydryl amino acid and the corresponding aldehyde compound by non-enzymatic ring-opening hydrolysis. $\mathrm{L}$-cysteine is required in the first of the two-step biosynthesis of tripeptide glutathione (GSH), ${ }^{5}$ which is a cellular protector. The use of monosaccharide aldoses as aldehyde donors

\footnotetext{
aDepartament of Chemistry, University of Lleida-Agrotecnio Center, Avda. Alcalde Rovira Roure, 191, E-25198, Lleida, Spain.E-mail: eras@quimica.udl.cat

${ }^{b} D B A R+D$ Tecnio Center, University of Lleida, Avda. Alcalde Rovira Roure, 191, E25198, Lleida, Spain

$\dagger$ Electronic supplementary information (ESI) available: Analytical data of yield, melting point, optical rotation, ${ }^{1} \mathrm{H}$ NMR, ${ }^{13} \mathrm{C}$ NMR and IR absortions. Spray-drying operation conditions on the outlet air temperature $\left(T_{\text {outlet }}\right)$, on the yield $\left(N_{\mathrm{p}}\right)$, moisture content, bulk density (BD), and solubility of the products. Percentage conversion table in the batch system. ${ }^{1} \mathrm{H}$-NMR spectra of D-galactose and D-glucose derivatives of sodium 1,3-thiazolidine-4-carboxylates before and after spray drying. See DOI: 10.1039/c5ra19880a
}

instead of formaldehyde to obtain 2-polyhydroxyalkyl-1,3thiazolidine-4-carboxylic acids has been proposed ${ }^{2,6}$ because they have the advantage that they do not deliver toxic compounds when the condensation is reversed by in vivo hydrolysis. Moreover, the presence of hydroxyl groups of carbohydrate moiety increases the solubility of these 2S-TCA derivatives in water. In general, these compounds show biological properties of interest. In this regard, they exert antitumor activity, ${ }^{7}$ show radioprotective properties, ${ }^{8}$ prevent the formation of cataracts, ${ }^{9,10}$ and inhibit lipid peroxidation. ${ }^{11}$ Furthermore, they are potential acetaminophen antidotes..$^{12,13}$ Moreover, the thiol group has the capacity to trap free radicals. ${ }^{14}$ The anti-oxidant properties of some 2S-TCA derivatives from various aldohexose in vitro models have also been reported. ${ }^{15}$

Strategies to prepare these compounds are based on mixing a solution of cysteine or cysteine hydrochloride and an aldopentose or aldohexose in an equal molar ratio in the presence of a base in a batch reactor. The concentration, ${ }^{3,16}$ excess of reactants, ${ }^{4}$ reaction temperature, base, and solvent used will determine the efficiency of the reaction. Methanol and also water have been used as solvents. When the latter is used, the products are recovered by crystallization by adding large amounts of ethanol to the solution. ${ }^{2}$ For the products synthesized by refluxing methanol, spontaneous 
precipitation occurs as the products are formed. However, recrystallization of TCAs results in a considerable loss of product. ${ }^{6}$ The use of a base in the reaction, generally pyridine, is not necessary but does increase the yield. Other bases used in this synthesis include potassium acetate ${ }^{17}$ and sodium bicarbonate..$^{2,18}$

In view of the economic interest of these compounds, mainly in their inclusion in agrochemicals, food, and feed additives, ${ }^{19-21}$ here we sought to optimize the production system. In this regard, we substituted the previous reaction methods based on batch systems for a continuous-flow system (CFS). Such systems have many advantages, such as efficiency, simplicity, facile automation, reproducibility, and process reliability by an accurate control of reaction conditions. ${ }^{22-25}$

To achieve our purposes, water and sodium hydroxide were used as the solvent and base, respectively, to obtain TCAs from formaldehyde and aldoses. Moreover, sodium hydroxide in equimolar amount with L-cysteine and aldoses was used in order to obtain the TCAs as sodium salts. The proposed process could be considered a suitable alternative to prepare pharmaceutical or food-grade products as it uses water as solvent and sodium hydroxide as base. To recover the product, instead of using large amounts of ethanol, which is expensive, we employed spray drying. Widely used for converting a broad range of solved products into powder form, this technique has many applications in the pharmaceutical, food, and agrochemical industries. ${ }^{26-29}$ Spray-dried powders are economical to produce compared to other processes such as freezedrying. ${ }^{30}$

In summary, aim of this work was the preparation of the salts of TCA and 2S-TCAs from formaldehyde and natural aldoses (D-glucose, D-galactose, D-mannose, D-xylose, D-ribose and D-arabinose) (Fig. 1). The syntheses were performed in a continuous-flow system reactor, and the products were then recovered by means of spray drying. The efficacy of the process was measured by the amount, stability and quality of the products recovered, on the basis of the specifications most commonly used in spray-dried powders, namely moisture content, bulk density, and solubility rate.
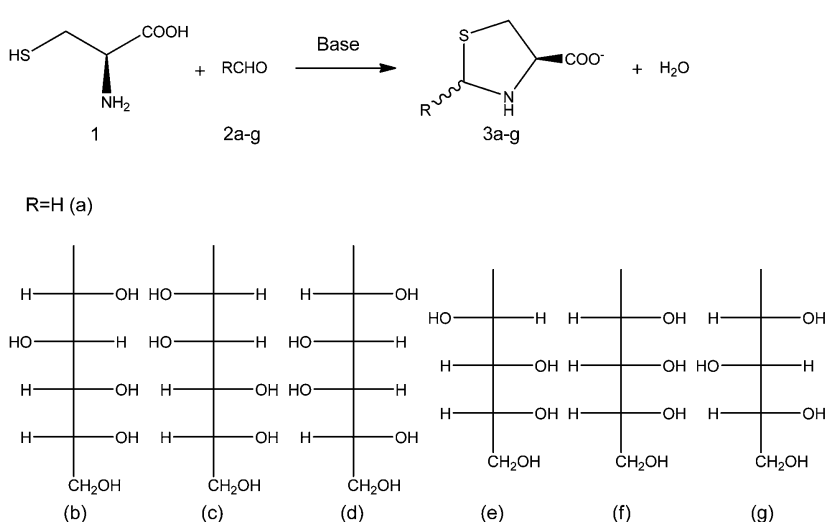

Fig. 1 (a): Formaldehyde, (b): D-(+)-glucose, (c): D-(+)-mannose, (d): $\mathrm{D}-(+)$-galactose, (e): $\mathrm{D}-(-)$-arabinose, $(\mathrm{f})$ : $\mathrm{D}-(-)$-ribose, and $(\mathrm{g})$ : $\mathrm{D}$ (+)-xylose.

\section{Experimental section}

\section{Materials}

L-Cysteine, formaldehyde, $\mathrm{D}-(+)$-glucose and $\mathrm{D}-(+)$-xylose were purchased from Sigma-Aldrich (Sigma-Aldrich Química, S.A., Madrid, Spain). D-(+)-mannose, D-(+)-galactose, D-(-)-arabinose, and D- $(-)$-Ribose were supplied by Acros (Madrid, Spain). Sodium hydroxide was obtained from Fischer (Madrid, Spain).

\section{Continuous-flow system reaction}

The continuous-flow system (FRX System, Syrris Ltd, Royston, UK) consisted of a $4 \mathrm{~mL}$ PTFE tube reactor $(0.8 \mathrm{~mm}$ inner diameter, $8 \mathrm{~m}$ total length) (Fig. 2). The tube reactor was placed on a digitally controlled RCT basic hotplate (IKA-Werke $\mathrm{GmbH}$ \& Co., KG, Staufen, Germany) with external Pt 100 sensor for optimum control of temperature. The L-cysteine and aldose solutions were placed separately in a ratio of $1: 1$ in $1000 \mathrm{~mL}$ borosilicate glass bottles with a screw cap. We slowly added 1.75 moles $(70 \mathrm{~g})$ of $\mathrm{NaOH}$ to a $1.75 \mathrm{M}\left(211.75 \mathrm{~g} \mathrm{~L}^{-1}\right)$ solution of L-cysteine in order to reach a $\mathrm{pH}$ of approximately 9.5. The corresponding solution of aldehyde was also $1.75 \mathrm{M}$ (52.5$315.3 \mathrm{~g} \mathrm{~L}^{-1}$ ). A mixing-T junction before the reactor was used to combine the reagent solutions. The reagents were pumped through the tube reactor at a constant flow rate of between 0.5 and $1 \mathrm{~mL} \mathrm{~min}^{-1}$. Reaction temperatures of $70{ }^{\circ} \mathrm{C}$ or $80^{\circ} \mathrm{C}$ were used. The product was collected in a bottle, which in turn was connected to a spray-drying system. Conversions were quantified by the ratio of the characteristic peak areas of products and reagents from the ${ }^{1} \mathrm{H}$ NMR spectra, which were recorded on a VARIAN 400 NMR spectrometer. For the mixtures of aldoses, in the simulation of hydrolyzed hemicelluloses, the global conversion was measured by ${ }^{1} \mathrm{H}$ NMR quantification of the L-cysteine that remained unreacted.

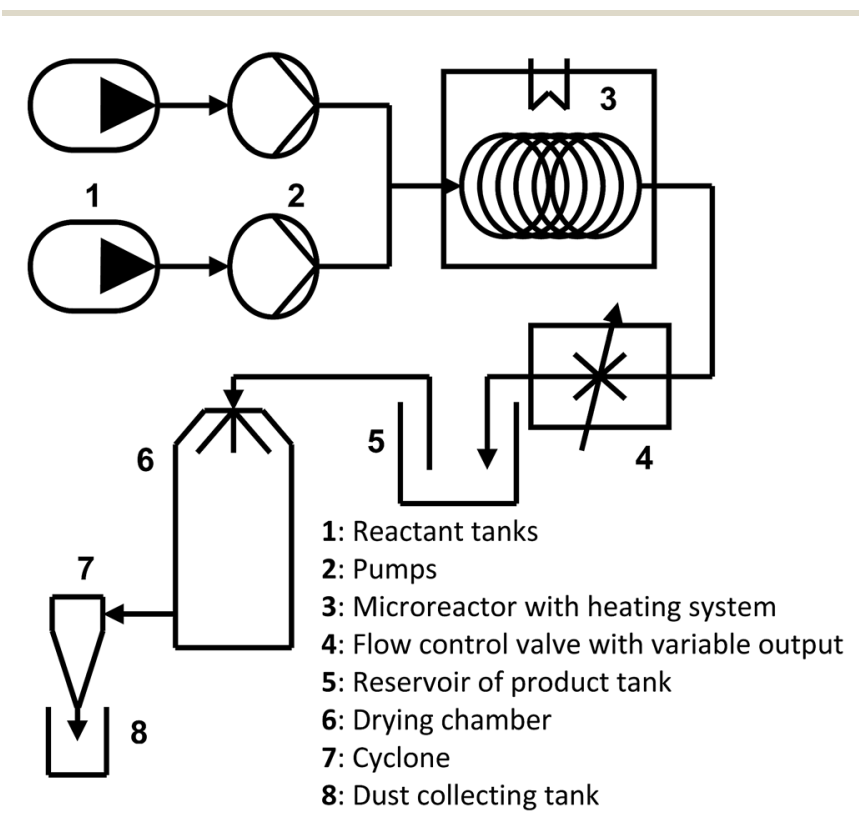

Fig. 2 Design of the continuous-flow system coupled to spray dryer. 


\section{Spray-drying system}

The resulting solution was collected in a $1 \mathrm{~L}$ bottle and injected into a Büchi B290 laboratory spray dryer (Flawil, Switzerland) to recover a powder. A peristaltic pump drove the solutions to the atomizer, and atomization was performed using a two-fluid nozzle (diameter $0.7 \mathrm{~mm}$ ), with a water evaporation capacity of $1 \mathrm{~L} \mathrm{~h}^{-1}$.

The following parameters were studied: feed rate; initial concentration of solid feed; flow rate of drying air (aspirator setting); and inlet air temperature. Three inlet air temperatures $\left(T_{\text {inlet }}\right)$, namely $125{ }^{\circ} \mathrm{C}, 140{ }^{\circ} \mathrm{C}$ and $160{ }^{\circ} \mathrm{C}$, were tested. The flow rate of drying air $\left(Q_{\mathrm{a}}\right)$ was 31 or $35 \mathrm{~m}^{3} \mathrm{~h}^{-1}$ (75 and $90 \%$ respectively of device capability). The feed rates tested were 3 $\mathrm{mL} \min ^{-1}(10 \%), 4.5 \mathrm{~mL} \min ^{-1}(15 \%)$ and $6 \mathrm{~mL} \min ^{-1}(20 \%)$. The flow rate of compressed air $\left(Q_{\mathrm{c}}\right)$ was maintained at $0.54 \mathrm{~m}^{3}$ $\mathrm{h}^{-1}$ in all experiments. Outlet air temperatures (Toutlet) were monitored continuously.

\section{Performance of spray dryer}

The powders of the products were collected mainly from cyclone. In addition, an extra powdered solid was recovered at the end of the process by gently sweeping the wall of the cylindrical part of the dryer chamber, as proposed in some spray-drying protocols. ${ }^{31,32}$ The dried particles were carefully recovered, weighed, and stored in a sealed glass vessel at room temperature. The yield $\left(N_{\mathrm{p}}\right)$ was calculated by the percentage of the ratio of the weight of the powder recovered in the spray dryer and the weight of dry solid reagents.

\section{Characterization of product powders}

Moisture content. The moisture content was determined by drying the powders at $70{ }^{\circ} \mathrm{C}$ in a vacuum oven until constant weight (less than $0.3 \%$ variation).${ }^{33}$ Moisture loss was expressed in terms of percentage of wet basis (wb) ( $\mathrm{kg}$ water $/ \mathrm{kg}$ wet material $\times 100$ ). Measurements were performed in triplicate.

Bulk density. The bulk density was calculated by dividing the mass of $2.00 \mathrm{~g}$ of powder measured in a $10 \mathrm{~mL}$ graduated test tube by the volume occupied in the tube. ${ }^{28,34}$ Measurements were performed in triplicate.

Solubility rate. The speed at which the powder dissolved was determined by adding $2.00 \mathrm{~g}$ of the material to $50 \mathrm{~mL}$ of distilled water at $26{ }^{\circ} \mathrm{C}$. The mixture was placed in a $100 \mathrm{~mL}$ low shape glass beaker with a Heidolph magnetic stirrer (no. 50382, MR 82, Heidolph Instruments GmbH \& Co. KG, Schwabach, Germany) at $892 \mathrm{rpm}$, using a magnetic $2 \mathrm{~mm} \times 7 \mathrm{~mm}$ stirring bar. The time required for the material to fully dissolve was recorded. ${ }^{34,35}$ Measurements were performed in triplicate.

Stability of the powder in water. Sodium salt solutions of TCA and 2S-TCAs in deuterium oxide $(100 \mathrm{mM})$ were analyzed by ${ }^{1} \mathrm{H}$ NMR at room temperature every five days over 30 days. Hydrolysis of the compounds was estimated as the percentage of the corresponding aldehyde and L-cysteine present in the solutions. The results were compared with those obtained from the acidic form of TCA and 2S-TCAs prepared with the classical method using methanol as solvent. ${ }^{2,6}$ Measurements were performed in triplicate.

\section{Results and discussion}

\section{Influence of $\mathrm{pH}$ on conversion percentage}

Initially, the conversion of TCAs in the continuous-flow system was evaluated using formaldehyde and $\mathrm{D}$-xylose. The reactor temperature was set at $70{ }^{\circ} \mathrm{C}$, and flow rates of $0.5 \mathrm{~mL} \mathrm{~min}{ }^{-1}$

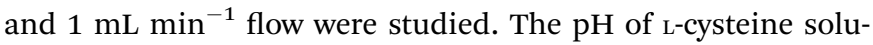
tion was adjusted with $8 \mathrm{M} \mathrm{NaOH}$ solution to various values ranging from 7 to 10 (Fig. 3). The conversion of 1,3-thiazolidin4-carboxylic acid was completed at $\mathrm{pH} 8$ to $\mathrm{pH} 10$ at both flow rates. Ratner and Clarke found that formaldehyde reacts with cysteine over a wide range of $\mathrm{pH}$ values to form thiazolidine-4carboxylic acid, the overall rate of formation being faster with increasing $\mathrm{pH} .^{3}$ The formation of 2S-TCA derived from D-xylose also increased at basic $\mathrm{pH}$. At $\mathrm{pH}$ 9, the conversion of this product reached 90\%; however, a further increase in $\mathrm{pH}$ did not improve the yield. As several authors have reported, the presence of a base was not required for TCA formation but did increase the overall yields. This observation may be attributable to displacement of the equilibrium of the amino group of cysteine to the non-protonated form, resulting in an increase in its nucleophilicity and thus favoring thiazolidine ring formation., ${ }^{1,36}$

Regarding the flow rates, at $70{ }^{\circ} \mathrm{C}$, the conversion was higher when the reactor worked with a low flow rate. However, very similar results were obtained with a flow rate of $1 \mathrm{~mL} \mathrm{~min}{ }^{-1}$, thus allowing an increase in productivity (Fig. 3). Along these lines, we sought to optimize the other reaction conditions, namely temperature and flow rate.

\section{Influence of temperature and pump flow rate}

Two temperatures $\left(70{ }^{\circ} \mathrm{C}\right.$ and $\left.80^{\circ} \mathrm{C}\right)$ and two pump flow rates

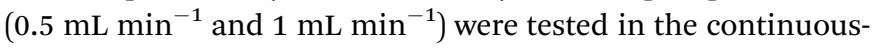
flow system for each aldehyde. The concentration of the solutions of L-cysteine, sodium hydroxide, and the corresponding aldehyde was $1.75 \mathrm{M}$. Table 1 shows the conversions achieved.

The conversions, ranging from 55 to $100 \%$, were greater when a reaction temperature of $80{ }^{\circ} \mathrm{C}$ rather than $70{ }^{\circ} \mathrm{C}$ was

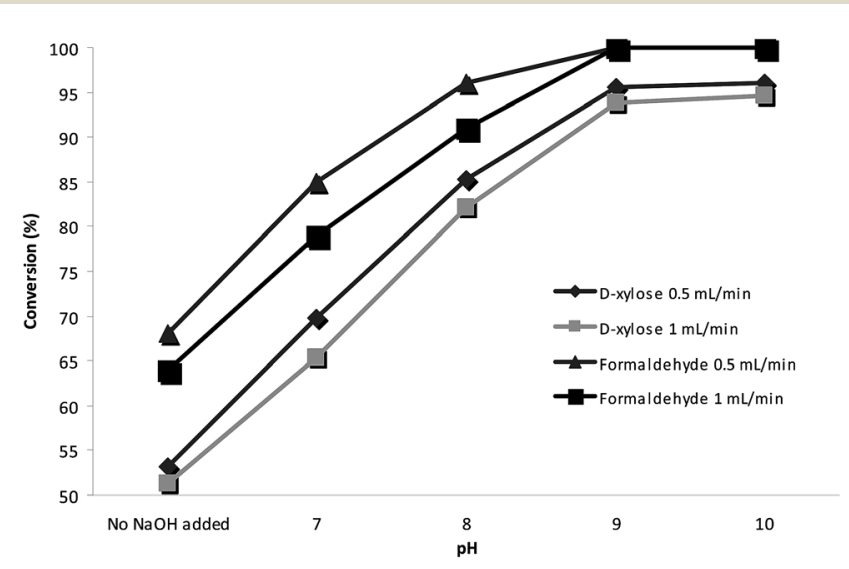

Fig. 3 Effect of the $\mathrm{pH}$ of the L-cysteine solution adjusted with $\mathrm{NaOH}$ and the flow rate on the conversion of $\mathrm{D}$-xylose and formaldehyde at $70{ }^{\circ} \mathrm{C}$. 
Table 1 Effect of the reactor temperature and the pump flow rate on aldehyde conversion ${ }^{a}$

\begin{tabular}{|c|c|c|c|c|c|c|}
\hline \multirow[b]{2}{*}{ Entry } & \multirow[b]{2}{*}{ Aldehyde } & \multicolumn{5}{|c|}{ Conversion (\%) } \\
\hline & & 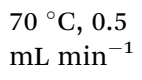 & $70{ }^{\circ} \mathrm{C}, 1 \mathrm{~mL} \mathrm{~min}{ }^{-1}$ & $80{ }^{\circ} \mathrm{C}, 0.5 \mathrm{~mL} \mathrm{~min}^{-1}$ & $80^{\circ} \mathrm{C}, 1 \mathrm{~mL} \min ^{-1}$ & Batch, $80{ }^{\circ} \mathrm{C}, 4 \mathrm{~h}$ \\
\hline $\mathrm{a}$ & Formaldehyde & 100 & 100 & 100 & 100 & 100 \\
\hline $\mathrm{b}$ & D-Glucose & 59 & 55 & 78 & 76 & 77 \\
\hline $\mathrm{c}$ & D-Mannose & 89 & 85 & 92 & 91 & 93 \\
\hline $\mathrm{d}$ & D-Galactose & 88 & 87 & 92 & 91 & 87 \\
\hline $\mathrm{e}$ & D-Arabinose & 90 & 89 & 92 & 92 & 86 \\
\hline $\mathrm{f}$ & D-Ribose & 93 & 91 & 94 & 92 & 91 \\
\hline$g$ & D-Xylose & 97 & 99 & 100 & 99 & 94 \\
\hline
\end{tabular}

${ }^{a}$ Conditions in batch reaction: $1.75 \mathrm{M}$ mixture solution of aldose : L-cysteine : NaOH $1: 1: 1$ molar ratio.

used. TCA formation from formaldehyde in a $1: 1$ molar ratio was $100 \%$ in all the conditions tested. The rapid formation of this product in batch reactors has been described to be the result of the high reactivity of formaldehyde. ${ }^{3}$ In all cases, the reaction with aldopentoses gave greater conversions than with aldohexoses for the conditions tested. D-xylose achieved the best conversions, achieving $100 \%$ conversion at a flow rate of $0.5 \mathrm{~mL}$ $\min ^{-1}$ at $80{ }^{\circ} \mathrm{C}$. D-glucose was the aldose with the lowest reactivity. In this regard, other studies also reported lower conversions when synthesising 2 S-TCAs with this aldose. ${ }^{17}$

This observation could be attributable to the fact that the conformational equilibrium of D-glucose is shifted mainly towards the ring structure of hemiacetal rather than for the open free carbonyl structure. ${ }^{37}$ However, an increase in conversion from 59 to $78 \%$ was achieved when the reactor temperature was set at $80{ }^{\circ} \mathrm{C}$. Concerning the pump flow rate,

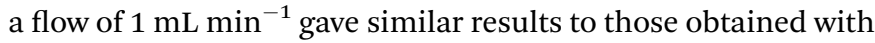
$0.5 \mathrm{~mL} \mathrm{~min}^{-1}$, as in the previous experiments.

\section{Mixtures of aldoses}

Mixtures containing aldohexoses and aldopentoses were prepared in various ratios at a final concentration of $1.75 \mathrm{M}$ (Table 2). These mixtures simulated the resulting mixtures of aldoses obtained from the hydrolysis of hemicelluloses. ${ }^{38,39}$ Sodium hydroxyde was added to a solution of L-cysteine (1.75 $\mathrm{M})$ to adjust the $\mathrm{pH}$ to 9.5 . The reactor temperature and pump flow rate were the same as those used in the previous experiments. Table 2 shows the conversions obtained for each mixture.

Mixtures of 2S-TCAs from the corresponding mixtures of aldoses were obtained in a similar percentage of conversions as in the experiments with individual aldoses (Table 2). Conversions varied from 75.5 to $92.5 \%$ depending on the reactor temperature and flow rate. For the four mixtures prepared, the highest conversions were achieved at $80{ }^{\circ} \mathrm{C}$ with a pump flow rate of $0.5 \mathrm{~mL} \mathrm{~min}^{-1}$. The mixtures formed by aldopentoses achieved greater conversion rates than those formed by aldohexoses or combinations of the same.

The mixtures that included $\mathrm{D}$-glucose registered the lowest conversions.

\section{Spray-drying performance}

The solutions of TCA and 2S-TCAs showing the best conversions in the continuous-flow system were subjected to spray drying. For each solution, three inlet temperatures $(125,140$ and $\left.160{ }^{\circ} \mathrm{C}\right)$ and three feed flow rates $\left(3,4.5\right.$ and $\left.6 \mathrm{~mL} \mathrm{~min}^{-1}\right)$ were tested. These two parameters together with the flow rate of drying air $\left(Q_{\mathrm{a}}\right)$ condition the air outlet temperature. Considering all these parameters the aspiration setting was adjusted depending on the product. The spray-drying operating conditions, the percentages of product recovered, and the characteristics of the powders produced are described in ESI. $\uparrow$ Table 3 summarizes the results using the best spray-drying operating conditions.

Table 2 Effect of temperature and flow rate on the conversion of aldose mixtures in the continuous-flow system reactor ${ }^{a}$

\begin{tabular}{|c|c|c|c|c|c|}
\hline Aldose mixtures (entry) & Aldose ratio & \multicolumn{4}{|l|}{ Conversion (\%) } \\
\hline $\mathrm{d}, \mathrm{b}, \mathrm{c}$ & $1: 1: 3$ & 85.2 & 83.3 & 86.1 & 84.6 \\
\hline$e, d$ & $1: 2$ & 90.8 & 89.5 & 92.8 & 92.2 \\
\hline $\mathrm{e}, \mathrm{g}$ & $1: 2$ & 92.1 & 88.2 & 93.3 & 92.5 \\
\hline
\end{tabular}

${ }^{a}$ Simulated hemicelluloses: b, c: glucomannan (D-glucose, D-mannose). d, b, c: galactoglucomannan (D-galactose, D-glucose, D-mannose). e, d: arabinogalactan (D-arabinose, D-galactose). e, g: arabinoxylan (D-arabinose, D-xylose). The L-cysteine : aldose ratio was maintained at $1: 1$. 
Table 3 Effect of the best spray-drying operating conditions on the outlet air temperature $\left(T_{\text {outlet }}\right)$ and on the yield $\left(N_{\mathrm{p}}\right)$, moisture content, bulk density (BD), and solubility of the product ${ }^{a}$

\begin{tabular}{lllllll}
\hline Entry & $\begin{array}{l}Q_{\mathrm{a}} \\
(\%)\end{array}$ & $\begin{array}{l}T_{\text {outlet }} \\
\left({ }^{\circ} \mathrm{C}\right)\end{array}$ & $\begin{array}{l}N_{\mathrm{p}} \\
(\%)\end{array}$ & $\begin{array}{l}\text { Moisture }^{\mathrm{a}} \\
(\%)\end{array}$ & $\begin{array}{l}\mathrm{BD}^{\mathrm{b}} \\
\left(\mathrm{g} \mathrm{mL} \mathrm{mL}^{-1}\right)\end{array}$ & $\begin{array}{l}\text { Solubility rate }^{\mathrm{a}} \\
(\mathrm{s})\end{array}$ \\
\hline $\mathrm{a}$ & 90 & 60 & 83.9 & $0.72 \pm 0.03$ & 0.23 & $85 \pm 3$ \\
$\mathrm{~b}$ & 90 & 85 & 79.5 & $0.77 \pm 0.02$ & 0.25 & $185 \pm 3$ \\
$\mathrm{c}$ & 90 & 56 & 79.1 & $0.80 \pm 0.04$ & 0.25 & $189 \pm 3$ \\
$\mathrm{~d}$ & 90 & 66 & 82.2 & $0.68 \pm 0.02$ & 0.23 & $179 \pm 3$ \\
$\mathrm{e}$ & 75 & 69 & 81.8 & $0.58 \pm 0.04$ & 0.26 & $159 \pm 4$ \\
$\mathrm{f}$ & 75 & 68 & 80.8 & $0.71 \pm 0.03$ & 0.28 & $161 \pm 4$ \\
$\mathrm{~g}$ & 75 & 67 & 81.6 & $0.53 \pm 0.03$ & 0.28 & $152 \pm 5$ \\
$\mathrm{~g}$ & 90 & 70 & 48.1 & $0.85 \pm 0.03$ & 0.29 & $167 \pm 3$ \\
$\mathrm{~b}, \mathrm{c}$ & 75 & 74 & 74.9 & $0.80 \pm 0.02$ & 0.29 & $183 \pm 3$ \\
$\mathrm{~d}, \mathrm{~b}, \mathrm{c}$ & 75 & 72 & 81.9 & $0.93 \pm 0.03$ & 0.28 & $181 \pm 4$ \\
e, d & 75 & 74 & 78.2 & $0.94 \pm 0.02$ & 0.28 & $174 \pm 4$ \\
$\mathrm{e}, \mathrm{g}$ & 75 & 75 & 77.4 & $0.60 \pm 0.05$ & 0.29 & $168 \pm 3$
\end{tabular}

${ }^{a} T_{\text {inlet }}: 160{ }^{\circ} \mathrm{C}$. Feed flow: $3\left(\mathrm{~mL} \mathrm{~min}^{-1}\right)^{\mathrm{a}}$ mean \pm standard deviation, ${ }^{\mathrm{b}}$ mean with a standard deviation less than 0.01 .

\section{Product recovery}

The product recovery or yield, expressed as a percentage, is of primary interest as it reflects the performance of the process. In this regard, we obtained recoveries ranging from 7.8 to $83.9 \%$. Low values indicate that a considerable amount of solids was deposited on the walls of the drying chamber. For all the atomized powders, an increase in product recovery was achieved for experiments conducted with an inlet air temperature of $160{ }^{\circ} \mathrm{C}$ and a pump flow rate of $3 \mathrm{~mL} \mathrm{~min}{ }^{-1}$. These parameters led to a decrease in residue accumulation on the wall of the drying chamber, thus implying an increase in product yield. According to several authors, recovery values exceeding $70 \%$ are not usual in spray-drying operations because of the difficulties in collecting small and low-weight particles. ${ }^{31}$ In our case, the yields achieved with some aldehydes reached $83 \%$. The sodium salt of thiazolidine-4-carboxylic acid showed the greatest recovery, ranging from 50.3 to $80.9 \%$. This result may be due to the nature of the spray-dried material. For the remaining 2S-TCAs, the yields varied from 7.8 to $82.2 \%$. However, we detected a difference in the yields of aldoses. In case of $\mathrm{D}$-xylose, an aspiration setting of $90 \%$ did not achieve the recovery values obtained with the other aldoses. In this case, the same conditions but with an aspiration of $75 \%$ allowed an increase in yield. This observation could be associated with a decrease in the flow rate of the drying air causing an increment in product residence time in the drying chamber, thus leading to a greater degree of moisture removal. Consequently, these products have a low moisture content, and the particles do not tend to stick together and therefore do not form wall deposits. An aspiration setting of $75 \%$ was maintained for the remaining aldopentoses, namely Darabinose and D-ribose. With respect to the mixtures of aldoses, recovery values ranged from 77.4 to $81.9 \%$. These results were obtained with an inlet temperature of $160{ }^{\circ} \mathrm{C}$ and a pump flow rate of $3 \mathrm{~mL} \min ^{-1}$ (10\% of the device capability). Fig. 4 shows the powder yield against inlet air temperature and pump flow rate for the sodium salt of TCA derived from D-glucose.

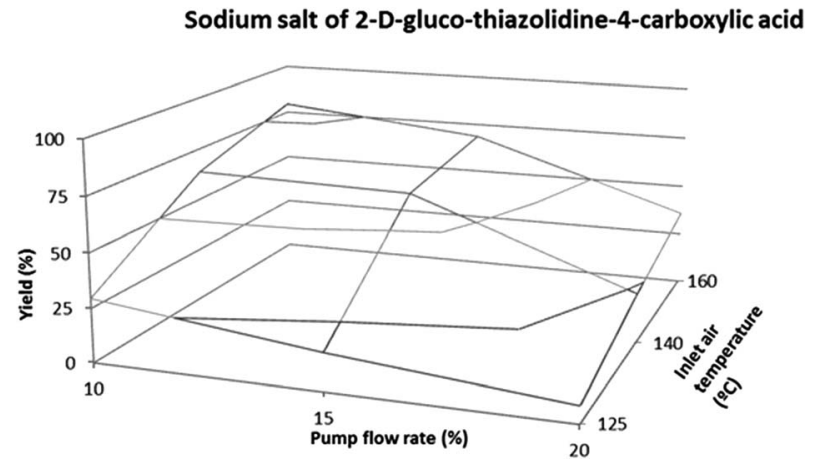

Fig. 4 Powder recovery as a function of inlet air temperature and pump flow rate for the sodium salt of 2-D-gluco-thiazolidine-4carboxylic acid.

\section{Outlet temperature}

The range of outlet temperatures varied from 41 to $80{ }^{\circ} \mathrm{C}$. Table 3 shows that outlet temperatures increased as the inlet air temperatures rose and also the flow rate of drying air.

This observation is consistent with the findings of other studies, ${ }^{\mathbf{4 0 , 4 1}}$ which reported an increase in outlet temperature in response to an increment in aspirator capacity. For the same compressed air flow rate, we found that a greater aspirator capacity increased outlet air temperature. The consequence was an enhanced yield because the powder was exposed to a high temperature for longer, thus improving drying efficacy.

\section{Powder properties}

The moisture content of the powders varied from 0.58 to $1.58 \%$. An increase in air inlet temperature is associated with a decrease in moisture content (ESI $\dagger$ ). For each inlet air temperature, powder moisture increased with an increase in the pump flow rate. This observation was similar for all the aldehydes. Generally, in a spray-drying system, the temperature of the exhaust air leaving the drying chamber determines the residual moisture of the powder. ${ }^{42,43}$ In these experiments, the values were very low and showed little variation, thus indicating complete drying.

Bulk density ranged from 0.23 to $0.35 \mathrm{~g} \mathrm{~mL}^{-1}$. For the sodium salt of thiazolidne-4-carboxylic acid, the values varied from 0.23 to $0.31 \mathrm{~g} \mathrm{~mL}^{-1}$, and from $0.23 \mathrm{~g} \mathrm{~mL}^{-1}$ for the sodium salts of galacto-thiazolidine-4-carboxylic acids to $0.35 \mathrm{~g} \mathrm{~mL}^{-1}$ for the sodium salt of 2-D-manno and 2-D-xylo-thiazolidine-4carboxylic acid. At a constant feed flow rate, an increment in the inlet air temperature led to a decrease in bulk density. This finding could be explained by faster evaporation rates and the products drying to a more porous structure. ${ }^{\mathbf{4 4 , 4 5}}$

Solubility rate in water ranged from 85 to $227 \mathrm{~s}$. In general, powders showed higher rehydration capacity with an increase in inlet air temperature. Low moisture content appears to be associated with rapid dissolution. ${ }^{34}$ Increasing the inlet air temperature produces an increase in particle size, thus accelerating rehydration. ${ }^{44}$ Large particles may sink, whereas small ones are dustier and generally float on water, thus 
allowing for uneven wetting and reconstitution. ${ }^{46}$ The rehydration capacity of the sodium salt of thiazolidine-4carboxylic acid ranged from 85 to $123 \mathrm{~s}$ and was higher than that of sodium salts of derivates from aldoses, which varied from 159 to $227 \mathrm{~s}$.

\section{Stability to hydrolysis in aqueous media}

Ferrari et al. ${ }^{47}$ described the great instability of the thiazolidine ring in the product derived from D-glucose, observing the presence of aldose after $24 \mathrm{~h}$ at $27{ }^{\circ} \mathrm{C}$ in acidic conditions. To describe and quantify the degradation of sodium salts of TCAs, freshly prepared solutions of the powders in deuterium oxide (10 mM) were analyzed by ${ }^{1} \mathrm{H}$-NMR every five days for one month at room temperature. The degradation results of the sodium salts of 2S-TCAs were compared with the corresponding ones of 2 S-TCAs obtained with the classical method. Fig. 5 and 6 show the percentage of dissociation of the products of TCAs and their corresponding sodium salts. TCAs were more unstable than the corresponding sodium salts prepared by the methodology described in this study. Both thiazolidine-4carboxylic acid and its sodium salt were remarkably stable, with no hydrolysis during the analysis. This observation is similar to that reported by Ratner and Clarke, who found that TCA was stable toward both acidic and alkaline media. ${ }^{3}$ However, 2S-TCAs synthesized from aldoses and the corresponding sodium salts were unstable and slowly decomposed on aging. Comparing 2S-TCAs and their corresponding sodium salts, the presence of cysteine and aldehydes was detected in the former after $24 \mathrm{~h}$, and the acids showed faster decomposition than the sodium salts. This result is in agreement with the reported findings concerning the sodium and potassium salts of similar compounds prepared by condensation of cysteine and aliphatic aldehydes.,48 Moreover, Roberts described that the decomposition of TCA derived from D-ribose in aqueous solution depended on the concentration and he observed a fast initial release of L-cysteine. ${ }^{49}$ In the present study, after one month, the percent of dissociation of 2S-TCAs ranged from 59.8 to $92.1 \%$, while the sodium salts varied from 13.6 to $17.7 \%$. Fig. 5 and 6 show that TCAs derived from aldohexoses were less stable than those obtained from aldopentoses. This difference was not observed when the products were in the sodium salt form.

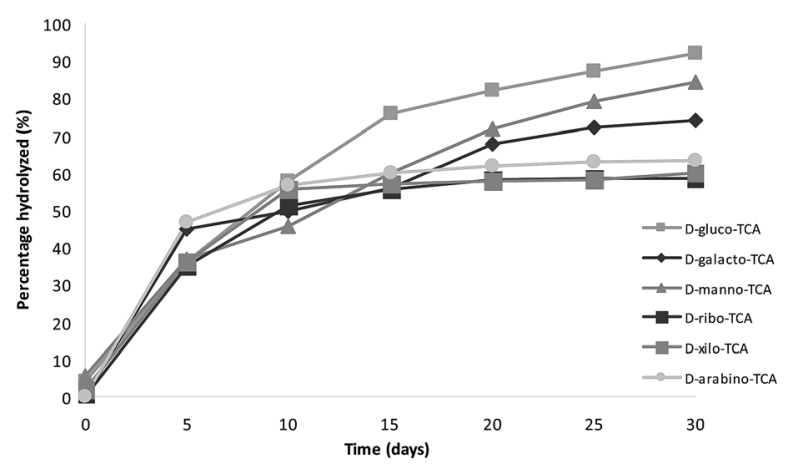

Fig. 5 Percentage of hydrolysis of the 2S-TCAs derived from aldoses.

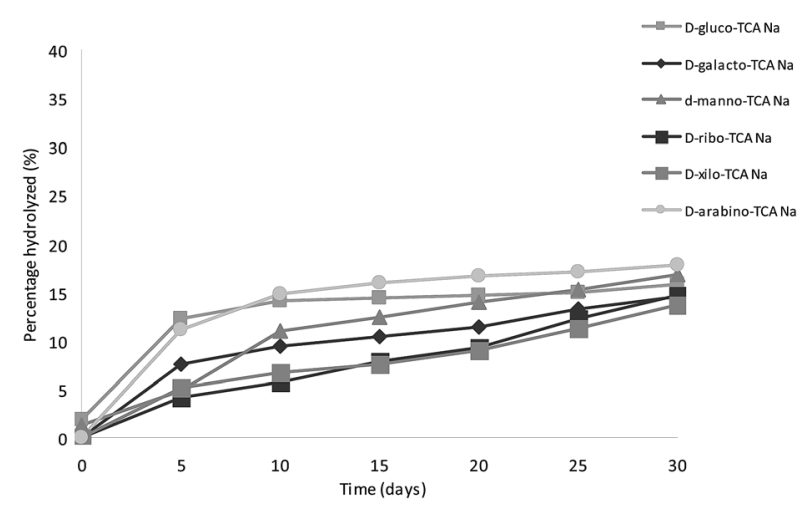

Fig. 6 Percentage of hydrolysis of the sodium salts of 2S-TCAs derived from aldoses.

\section{Conclusions}

Here we prepared the sodium salts of 1,3-thiazolidine-4carboxylic acid from formaldehyde and 2-substituted 1,3thiazolidine-4-carboxylic acids from three aldohexoses and three aldopentose in high conversions and yields in powder form. The methodology, based on combining a continuous flow reaction and a spray-drying system, allows the accurate control of the process and facilitates scaling. This approach, combined with the use of water and sodium hydroxide as solvent and base, respectively, allows the preparation of products in compliance with food-grade quality with a low environmental impact. Moreover, the approach does not involve the use of alcohols or organic bases, thus improving production costs.

When applied to mixtures of aldoses, this process also yields satisfactory results, thus opening up the possibility to exploit the hydrolysates of hemicelluloses.

The main characteristics of the powder products were determined, finding that the sodium salts of TCAs have some advantages. Our experiments reveal that the salts are easier to recover, highly soluble in water, more stable to hydrolysis in aqueous solution than the corresponding acidic forms, and easily convertible to an acidic form, by acid addition, if required.

\section{Acknowledgements}

The authors are grateful to Ministerio de Educación, Cultura y Deportes of Spain (FPU program) for the UdL grant of Olalla Novo Fernández (AP2010-1806). We also thank Tania Yates for the proofreading of the manuscript.

\section{Notes and references}

1 M. P. Schubert, J. Biol. Chem., 1936, 114, 341-350.

2 M. P. Schubert, J. Biol. Chem., 1939, 130, 601-603.

3 S. Ratner and H. T. Clarke, J. Am. Chem. Soc., 1937, 59, 200206.

4 I. R. Schmolka and P. E. Spoerri, J. Org. Chem., 1957, 22, 943946. 
5 J. Viña, R. Hems and H. A. Krebs, Biochem. J., 1978, 170, 627630.

6 J. C. Roberts, H. T. Nagasawa, R. T. Zera, R. F. Fricke and D. J. W. Goon, J. Med. Chem., 1987, 30, 1891-1896.

7 Y. Zhang, C. Sun, Z. Chen, X. Li and R. Wang, Yiyao Gongye, 1988, 19, 152-154.

8 J. C. Roberts, K. E. Koch, S. R. Detrick, R. L. Warters and G. Lubec, Radiat. Res., 1995, 143, 203-213.

9 W. B. Rathbun, C. E. Killen, A. M. Holleschau and H. T. Nagasawa, Biochem. Pharmacol., 1996, 51, 1111-1116.

10 W. B. Rathbun, H. T. Nagasawa and C. E. Killen, Exp. Eye Res., 1996, 62, 433-441.

11 L. Wlodek and J. Czubak, Pol. J. Pharmacol. Pharm., 1989, 41, 369-375.

12 H. T. Nagasawa, D. J. W. Goon, W. P. Muldoon and R. T. Zera, J. Med. Chem., 1984, 27, 591-596.

13 J. C. Roberts, R. L. Charyulu, R. T. Zera and H. T. Nagasawa, Pharmacol. Toxicol., 1992, 70, 281-285.

14 R. E. Durand, Int. J. Radiat. Oncol., Biol., Phys., 1984, 10, 1235-1238.

15 Y. Yan, L. Wan-Shun, H. Bao-Qin and S. Hai-Zhou, Nutr. Res., 2006, 26, 369-377.

16 W. C. Hess and M. X. Sullivan, J. Biol. Chem., 1937, 121, 323329.

17 J. Radomski and A. Temeriusz, Carbohydr. Res., 1989, 187, 223-237.

18 B. Refouvelet, N. Pellegrini, J.-F. Robert, G. Crini, O. Blacque and M. M. Kubicki, J. Heterocycl. Chem., 2000, 37, 1425-1430.

19 D. Bagley, S. Momii, S. Nagasawa and H. T. Nagasawa. US Pat., 2015/0023941, 2015.

20 M. Bonfiglioli and L. Filippini, WO Pat, 03/079790, 2003.

21 K. B. de Roos, in Flavor Precursors, ed. R. Teranishi, G. R. Takeoka and M. Güntert, American Chemical Society, Washington DC, 1st edn, 1992, vol. 490, ch. 16, pp. 203-216.

22 E. Yara-Varón, J. Eras Joli, M. Balcells, M. Torres and R. Canela-Garayoa, RSC Adv., 2012, 2, 9230.

23 G. Jas and A. Kirschning, Chem.-Eur. J., 2003, 9, 5708-5723.

24 K. F. Jensen, Chem. Eng. Sci., 2001, 56, 293-303.

25 P. D. I. Fletcher, S. J. Haswell, E. Pombo-Villar, B. H. Warrington, P. Watts, S. Y. F. Wong and X. Zhang, Tetrahedron, 2002, 58, 4735-4757.

26 R. Vehring, Pharm. Res., 2008, 25, 999-1022.

27 C. Vega, H. D. Goff and Y. H. Roos, J. Food Sci., 2006, 70, 244-251.
28 A. M. Goula and K. G. Adamopoulos, Innovative Food Sci. Emerging Technol., 2010, 11, 342-351.

29 A. Soottitantawat, H. Yoshii, T. Furuta, M. Ohkawara and P. Linko, J. Food Sci., 2003, 68, 2256-2262.

30 D. Knorr, Trends Food Sci. Technol., 1998, 9, 295-306.

31 B. R. Bhandari, N. Datta and T. Howes, Drying Technol., 1997, 15, 671-684.

32 B. Adhikari, T. Howes, B. J. Wood and B. R. Bhandari, J. Food Eng., 2009, 94, 135-143.

33 P. G. Goose and R. Binsted, inTomato paste, puree, juice and powder, Food Trade Press, London, 1st edn, 1964, pp. 124126.

34 A. M. Goula and K. G. Adamopoulos, J. Food Eng., 2005, 66, 35-42.

35 A. H. El-Tinay and I. A. Ismail, Acta Aliment., 1985, 14, 283295.

36 R. Bognár, L. Somogyi and Z. Györgydeák, Justus Liebigs Ann. Chem., 1970, 738, 68-78.

37 H. F. Bunn and P. J. Higgins, Science, 1981, 213, 222-224.

38 P. Gallezot, Chem. Soc. Rev., 2012, 41, 1538-1558.

39 E. Sjöström, in Wood Chemistry: Fundamentals and Applications, Academic Press, San Diego, 2nd edn, 1993, vol. 3, pp. 51-70.

40 P. Tewa-Tagne, G. Degobert, S. Briancon, C. Bordes, J.-Y. Gauvrit, P. Lanteri and H. Fessi, Pharm. Res., 2007, 24, 650-661.

41 A. M. Goula and K. G. Adamopoulos, J. Food Eng., 2005, 66, 25-34.

42 K. Masters, Spray drying handbook, George Godwin Ltd., London, 1979.

43 E. M. Cook and H. D. Dumont, Process Drying Practice, Mcgraw-Hill, New York, 1991.

44 D. E. Walton, Drying Technol., 2000, 18, 1943-1986.

45 G. R. Chegini and B. Ghobadian, Drying Technol., 2005, 23, 657-668.

46 N. N. Potter, Food Science, Avi Publishing Company, Westport, 1968.

47 E. Ferrari, R. Grandi, S. Lazzari, G. Marverti, M. C. Rossi and M. Saladini, Polyhedron, 2007, 26, 4045-4052.

48 I. T. Strukov, Zh. Obshch. Khim., 1952, 22, 521-527.

49 J. C. Roberts and D. J. Francetic, Med. Chem. Res., 1991, 1, 213-219. 